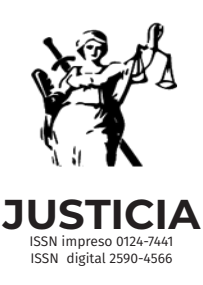

\title{
Marco jurídico de la paz en Colombia. Una revisón sistemática y crítica
}

\section{Legal framework of peace in Colombia. A systematic and critical review}

\author{
(iD) \\ Judith J. Hernández García de Velazco \\ Universidad de la Costa CUC \\ lasanas23@gmail.com
}

\author{
Andreina Cerpa Muñoz \\ Universidad de la Costa CUC \\ andreina13cerpa@gmail.com
}

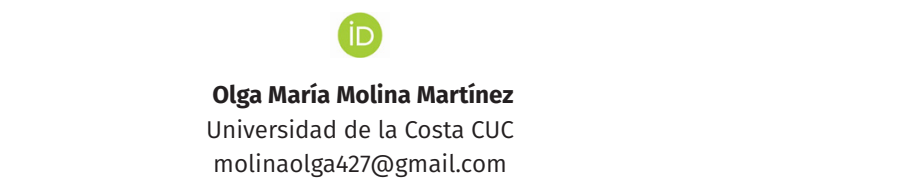

Olga María Molina Martínez

molinaolga427@gmail.com

Recibido: 24 de Agosto de 2020 / Aceptado: 30 Agosto de 2020 https://doi.org/10.17081/just.25.38.4485

\begin{abstract}
Resumen
La presente disertación, se orienta hacia el análisis sobre el proceso de paz en Colombia según el ordenamiento colombiano y desde un contexto histórico desde el cual se consideran los aspectos y factores que han hecho perdurar el conflicto. Los conflictos en Colombia se remontan desde los años de la independencia de Suramérica, en las cuales se luchó por la libertad frente al yugo español, sin embargo, las olas de violencia no cesaron porque hasta siglos después perduran con enfrentamientos intestinos, ya no por temas de territorialidad extracontinental sino por desacuerdos en ideológicos entre los partidos liberales y conservadores. Así se originaron grupos de enfrentamientos en la zona rural de Colombia, en la búsqueda del poder, del dominio, en un inicio en nombre de la justicia y la inclusión, entre otros, pero luego por la consecución de objetivos mediados por posiciones políticas extremas. Así como el conflicto también ha habido intentos de arreglos para la consecución de la paz, en tal sentido el acuerdo del proceso iniciado en 2012 hasta su firma en 2016 y posterior aplicación hasta el 2020. Estas dinámicas fueron examinadas desde una perspectiva cualitativa, de revisión sistémica documental, bibliográfica y de análisis, por lo que se infirió, entre algunas conclusiones; que se deben definir acuerdos con responsabilidad penal de los que han delinquido en contra de las personas, bienes públicos, generando diversos actos de violencia, privación de libertad y asesinatos masivos.
\end{abstract}

Palabras ClaVe: conflicto, marco jurídico, paz en colombia y proceso.

\begin{abstract}
This dissertation is oriented to the analysis of the peace process in Colombia according to the Colombian order and from a historical context in which the aspects and factors that have made the conflict endure are considered. The disputes in Colombia date back to the years of the independence of South America, in which freedom was fought for freedom from the Spanish yoke, however, the waves of violence did not cease because until centuries later they persist with internal confrontations, no longer for issues of extracontinental territoriality but because of ideological disagreements between the liberal and conservative parties. Thus, groups of fights arose in rural Colombia, in the search for power, dominance, initially in the name of justice and inclusion, among others, but later for the achievement of objectives mediated by extreme political positions. As well as the conflict, there have also been attempts to make arrangements to achieve peace.
\end{abstract}


In this sense, the agreement of the process started in 2012 until its signature in 2016 and subsequent application until 2020. These dynamics were examined from a qualitative perspective, of revision documentary, bibliographic and analytical systemic, for which it was inferred, among some conclusions. Those agreements should be defined with criminal responsibility for those who have committed crimes against people, public property, generating various acts of violence, deprivation of liberty, and mass murder.

KEYWORDS: conflict, legal framework, peace in Colombia and process.

Como Citar: Hernández García de Velazco, J. J., Cerpa Muñoz, A., \& Molina Martínez, O. M. (2020). Marco jurídico de la paz en Colombia. Una revisón sistemática y crítica. Justicia, 25(38), 232-242. https:// doi.org/10.17081/just.25.38.4485

\section{Introducción}

En esta disertación, se analizará el proceso de restitución de la paz en Colombia, estudiando sus implicancias históricas que le caracterizan como un tema de relevancia, dado que desde su configuración es evidente que el país ha experimentado relevantes hechos de violencia que la han marcado como nación, estas circunstancias le han influenciado en su desarrollo social e incluso económico, estos actos de violencia tienen una razón específica y un trasfondo en la lucha por los derechos fundamentales frente al conflicto que se ha producido.

En tal sentido, se puede definir dicha violencia, como una fuerza o energía drástica que generalmente es aplicada en casos extremos a ciertos actores sociales, con el fin de que actúen o dejen de actuar y más bien se ajusten a los intereses de esos grupos violentos.

En ese mismo orden de ideas, como lo expresan, Cerpa \& Molina (2019:11), "se entiende que la utilización de esa fuerza ha de aparecer bajo control en cualquier forma de sociedad, de manera que constituya un foco central, pero oculto, en la estructura de cualquier organización social, esta violencia puede tener efectos negativos como positivos, en cuanto a que generalmente se usa para la defensa de una causa, de la protección de un derecho al frente de un abuso de poder, por lo que representa un aspecto positivo, por otra parte, también es considerado negativo si se usa para tomar el control y el abuso de poder.

Necesariamente, en esta investigación se analizó la evolución que ha tenido la paz en el ordenamiento colombiano a través de la revisión bibliográfica-documental y del análisis sistematizado del contenido de diferentes corpus históricos y normativos, que explican la evolución del proceso de paz en Colombia, pero siempre en el entendido que Colombia ha experimentado muchas dificultades durante su proceso de restablecimiento de la paz y continua siendo un proceso en transición.

\section{Metodología}

Se realizó una revisión sistemática de documentos donde se trataron temas relacionados el marco jurídico de la paz en Colombia, evolución de la paz y los acuerdos de paz. Revisión Sistemática de aquellos artículos de aspectos cualitativos de estudios relacionados con las categorías de análisis.

Con el objetivo de resumir la información existente respecto de un tema en particular que sea de interés a los investigadores; la dinámica de la RS está basada en la recolecta de toda la información posible sobre el asunto de interés; posteriormente ser analizados, y por último compartir la evidencia que aportan con la de otros similares. Se aplicó, específicamente para esta investigación, en el análisis para el marco 
jurídico de la paz en Colombia, evolución de la paz y los acuerdos firmados en el año 2016.

Como criterio de aportación se asume que el proceso de paz ha ido ganando confianza a nivel nacional e internacional; ha hecho ruptura en el escepticismo inicial en torno del mismo, ha ido captando una mayor sintonía con el mismo por parte de distintos sectores de la sociedad civil, ha generado políticas públicas para la paz; ha logrado el diálogo y en algunos casos la articulación de distintos sectores de la sociedad civil, y muy importante ha posicionado la paz en la agenda pública del Estado y en la privada de los distintos sectores relevantes: la academia, la empresa privada, y los medios de comunicación, entre otros.

Como cultura para la paz en Colombia, es una aproximación desde las políticas públicas generadas para la consecución de la convivencia pacífica y su articulación en la praxis social con los valores de la cultura democrática en Colombia, desde los cuales se viabiliza, como propósito, el proceso de paz con las FARC iniciado en septiembre del 2012 con los diálogos que se desarrollaron en Cuba, pero exclusivamente a través de las políticas públicas que se derivan del mismo (2012-2020), y que a continuación se identifican para precisar los mecanismos políticos que impulsan la Convivencia pacífica. (Cerpa \& Molina, 2019).

Análisis de datos: La información analizada se estructuró en dos categorías: evolución de la paz en Colombia, y marco jurídico de la paz.

Del conjunto de estudios analizados se extrajo la información pertinente y las consideraciones exactas para la realización del artículo, de igual manera la necesidad de nuevas investigaciones, para una mejora en la intervención del marco jurídico de la paz en Colombia.

\section{Discusión-Resultados}

\section{Evolución de la paz en Colombia desde la discusión teórica y normativa}

Desde esta perspectiva, se asume la paz como un derecho humano. Para entender la paz como un derecho humano, es importante considerar que la humanidad en su generalidad ha recorrido un largo camino de guerras, conflictos, controversias, pasando por asesinatos masivos y el exterminio de personas por razones de diversas índoles, de igual manera en sus trances las sociedades, gobiernos, ciudadanos, han intentado de manera positiva resolver los conflictos en esfuerzos locales, regionales e internacionales, haciéndose énfasis para su concesión, por ejemplo, en la declaración universal de los derechos humanos, como una declaración de paz mundial.

En el mismo orden de ideas, el derecho humano para la paz, desde la perspectiva crítica del PNUD (2011), invita a los ciudadanos a reconocerse como tales, teniendo en cuenta su dignidad como ser humano y por consiguiente sus necesidades, el desarrollo de capacidades y potencialidades en el marco de una sociedad inclusiva y respetuosa marcada por la diferencia y pluralidad del ser humano, planteándose horizontes de vida que tengan altas aspiraciones de felicidad y desarrollo de su autonomía, en condiciones de florecimiento y seguridad humana, es decir lograr condiciones y medios para preservar el núcleo central de todas las vidas contra riesgos graves y previsibles.

De igual manera, en el PNUD con los Objetivos de Desarrollo Sostenible también conocidos como Objetivos Mundiales (2020), en particular con el objetivo No. 16 de Paz, justicia e instituciones sólidas, se le da un carácter global al tema de la paz duradera y sostenible. En cualquier contexto se hace un tema estratégico para el interés humano. 
Desde un análisis histórico y contextualizado, expresan Cerpa y Molina (2019), en ese ámbito social, quienes sufrieron directamente la violencia del conflicto armado buscan un proceso especial que implemente justicia, pero una justicia que satisfaga las necesidades de ecuanimidad y aspirando que el Estado colombiano desde su perspectiva jurídica del derecho a la paz, llegue a un común acuerdo en su mayoría, bajo el marco legal que impone la constitución a la hora de protección de derechos.

Desde esa evolución histórica jurídica, como un derecho para la paz, es preciso afirmar que a lo largo del tiempo Colombia ha logrado superar muchas de sus dificultades y problemas de violencia que hace años se perpetuaban, y hoy muchos quedan como memorias históricas; producto de los procesos anteriores de negociaciones que dieron con las nuevas regulaciones contenidas en la Constitución de 1991, y donde el Estado colombiano en la búsqueda por proteger a sus ciudadanos desde la Constitución política de 1991, estableció entonces el Estado social de derecho como una forma de regular derechos y deberes de forma efectiva e incluyente.

Tal cual lo expresan; Meléndez, Paternina y Velázquez (2018:58): “Entendiendo que la paz también puede derivarse de la guerra y de conflictos, es necesario que, para mantenerse en el tiempo, el sistema político debe reconocer los derechos participativos de cualquier ciudadano".

Coinciden Hernández, Chumaceiro, Zirritt y Acurero (2018), cuando afirman; que la paz requiere de la democracia como sistema político nato, pero adicionalmente se debe vivir la paz, como un proceso estructural de fondo que involucra lo social, político y económico. Todo cual ha determinado redefiniciones de las relaciones del Estado con la sociedad a través de diferentes políticas públicas auspiciadas desde las perspectivas de cada gobierno para la construcción de la paz social.

En consecuencia, el derecho humano a la paz es un derecho cuya responsabilidad le corresponde al Estado, pero con la activación de la ciudadanía en la continua vigilancia del cumplimiento de los deberes del Estado y la exigibilidad de nuevos derechos relacionados con la paz permanente, el goce y el disfrute de estos a plenitud en el marco de la emancipación de condiciones adversas al desarrollo humano, por ello el derecho a la paz es una construcción permanente de luchas y acuerdos, de compromisos y deberes entre los diversos estamentos de la sociedad y el Estado. En consecuencia, la convivencia es una necesidad vital para el conglomerado social, vivir en un entorno pacífico y sin violencia, es un derecho prioritario. Así como la obligación del Estado para preservar la paz e incluso reparar a víctimas de conflictos. (Trejos, Badillo \& Irreño, 2019; Narvaez, Matzumoto, Mira \& Zuñiga, 2019; García, 2019)

Por lo antes mencionado, hay que contextualizar que, a lo largo de la historia en Colombia desde su inicio como civilización no modernizada, asociada por las agrupaciones indígenas, así como en su tránsito durante la colonización española, y posterior con la independencia del yugo imperialista ibérico, hasta el S. XXI, su territorio se ha caracterizado por enfrentar la violencia como una secuela predominante en la sociedad.

Asimismo, la Constitución Política de Colombia del año 1991, fue producto del resultado de algunos procesos de negociación, con grupos armados al margen de la ley debido al descontento generalizado de una sociedad, que sentía que el marco constitucional vigente estaba lejos de responder a las necesidades sociales de la nación. Este instrumento jurídico, conocido como Constitución para la Paz, hizo referencia a la Paz en diversos artículos y desde diferentes ópticas. Donde se habló en el Preámbulo de la Paz como valor, en su Artículo 22, de la Paz como derecho fundamental, y la Paz como deber constitucional en el Artículo 95.

A esto se suma (Cerpa y Molina, 2019), la jurisprudencia de la Corte Constitucional de Colombia, que asume la Paz desde una polisemia amplia, como el estado ideal y como principio desde la perspectiva de 
derecho fundamental, asi como de derecho colectivo, con lo cual la ausencia de un concepto definitivo lleva a enormes dificultades en la comprensión del concepto y la reclamación judicial de las garantías que del mismo se podrían desprender.

Es así como la jurisprudencia constitucional en Colombia asume expresiones según sean los sujetos involucrados, las situaciones en conflicto y los mecanismos para solucionarlos, por lo tanto, evaluar estos conceptos permite un acercamiento dogmático que la Constitución Política de 1991, refiere y, por ende, lo que las autoridades y los particulares deben hacer en relación con este concepto. En tal sentido, las dificultades existentes en el país se incrementan cuando ocurre un proceso de negociación con un grupo armado ilegal, para introducirle un contexto normativo para la paz y donde el concepto de Paz como lenguaje sea un elemento más de la cotidianidad. Para tal fin se generó la necesidad de adelantar un estudio sobre, el significado dogmático del concepto Paz en la Constitución Política de Colombia de 1991, especial pero no únicamente desde la jurisprudencia de la Corte Constitucional de Colombia, sino partiendo de los fundamentos y perspectivas históricas jurídicas, que subyacen los deferentes pronunciamientos judiciales, para un acercamiento con unos elementos medulares que sirvan de consenso cuando el concepto de paz se utilice. (Cerpa y Molina, 2019),

En términos concretos la Paz es un derecho fundamental cuya protección no se puede equiparar con otros derechos de contenido hermenéutico menos problemático, por ser un evento de un perjuicio irremediable y ligado con el contenido de otros derechos puede ser objeto de protección, incluso mediante la acción de tutela histórica jurídica del derecho a la paz, garantizado por el estado colombiano. Además, la presente investigación en su análisis del derecho a la paz, parte como referencia de una categorización de la Constitución, cuyo fin es tratar de evidenciar si el derecho a la paz es una norma programática, normativa o semántica. Implica la revisión del derecho a la paz desde una perspectiva doctrinal y jurisprudencial, que marque su uso en diferentes contextos de la nación colombiana, donde el derecho a la paz tiene una significación bastante particular como consecuencia debido de la larga data del conflicto armado.

\section{Marco jurídico de la paz en Colombia}

El Congreso colombiano, en un intento de facilitar la negociación con las FARC-EP inscribiéndola en un esquema de justicia transicional, aprobó en julio de 2012 una reforma de la Constitución mediante el denominado "Marco Jurídico para la Paz"4 . A pesar de las críticas que ha recibido la aprobación de este Marco5, supone un intento, no exento de riesgos y contradicciones, de sentar las bases para una salida política al conflicto armado interno que ha asolado a Colombia desde hace más de cincuenta años y para la garantía de los derechos de las víctimas (Orozco, 2012). Para el 28 de agosto de 2013, la Corte Constitucional ha declarado exequible el Marco Jurídico para la Paz, lo que supuso un apoyo decidido a los lineamientos establecidos por el Gobierno de Juan Manuel Santos para alcanzar la paz con los grupos guerrilleros alzados en armas contra el Estado. Como señala el artículo 1 de este Marco, los instrumentos de justicia transicional "Garantizarán en el mayor nivel posible los derechos de las víctimas a la verdad, la justicia y la reparación".

\section{Mecanismos jurídicos que regulan la paz en Colombia}

Según Rodríguez, L (2014), estos mecanismos se definen como instrumentos jurídicos impulsados por el estado a fin de controlar o evitar un conflicto armado que pueda surgir dentro del país, estas herramientas se crean para otorgar justicia y eliminar los desacuerdos que se generan, entre los que se puede mencionar 
la Ley 975 de 2005, que ha sido importante ya que por medio de ésta se logró desintegrar un grupo de miembros de las autodefensas unidas de Colombia de alrededor 35.299 miembros, aunque a su vez presentaron dificultades debido a que el Estado quería condenar a todos sus miembros, y procedió a investigarlos individualmente, esto resultó en un fracaso porque no obtuvieron más de 15 sentencias condenatorias durante los ocho años siguientes al inicio del proceso.

Por medio de la Ley mencionada se elimina el reconocimiento político para todas las organizaciones armadas, y eliminando el poder de porte de Armas a las personas que por medio de actos probados hayan cometido asesinatos, secuestros, y cualquier acto atroz en perjuicio de la integridad del individuo. El presente marco normativo es denominado también Ley de Justicia y Paz, el cual fue aprobado para integrar a la sociedad a todos los miembros de grupos armados, con la condición de colaborar con la justicia, con el restablecimiento de la paz en la nación.

De acuerdo con Estrada (2019), en el propósito de recuperar la paz en Colombia y finiquitar el conflicto, se han decretado: (L.1448/2011) destinada básicamente a atender, asistir y reparar de forma integral a las víctimas del conflicto armado; para ello se dictan sentencias contra los responsables del hecho ilícito, se imponen medidas cautelares, entre otras decisiones judiciales en procura de reestablecer los derechos de la persona vulnerada. Posteriormente, con el propósito de que se facilitara y asegurara la implementación y desarrollo de las normas contempladas en el Acuerdo final, se concibió el fast track o procedimiento expedito, lo cual se concertó entre el Gobierno y las FARC. Esto venía a darle a esta organización guerrillera mayores garantías de cumplimiento. Aunado a ello, con (L.1820/2016), que versa sobre la amnistía, indulto y tratamientos especiales, era necesaria para que las FARC dejaran las armas y pasara a hacer vida política en el marco de las leyes.

Esta ley, (L.1820/2016), contempla el delito político y delitos conexos realizados durante una rebelión, y así se sustenta con los límites establecidos por el Estatuto de Roma, estableciendo así el Acuerdo de Paz que en los casos de delitos de lesa humanidad, genocidio, reclutamiento de menores, privación de libertad, toma de rehenes, desapariciones forzadas, ejecuciones extrajudiciales, acto carnal violento y otras formas de violencia sexual, sustracción de niños, desplazamiento forzado, entre otros actos de esta índole, no habría ninguna amnistía, indulto o cualquier otro beneficio equivalente. Vale acotar que sobre esta ley se pronunció la Corte Constitucional con sentencia (C. Const. Sentencia T-007/2018) de 1 de marzo la cual establecía que el reclutamiento de menores de 18 no sería considerado para amnistía debido al Protocolo Facultativo a la Convención del Derechos del Niño vigente en Colombia; pronunciamiento que obedece a que la ley establecía como delito no considerado para amnistía el reclutamiento de niños, pero menores de 15 años.

Esta ley (L.1820/2016), fue reforzada con la aprobación del decreto 277, el cual establece un procedimiento para su implementación efectiva. El propósito del decreto era acelerar la ley en los aspectos procesales, pues su implementación en su entrada en vigor había sido muy lenta, pues había una resistencia por parte de los jueces de la justicia ordinaria a aplicarla (Estrada, 2019)

Si bien se han manifestado críticas contra dicha ley, se puede alegar que en buena medida son infundadas pues Colombia cuenta con un historial de leyes de amnistía en las últimas décadas, como la (L.35/1982), la (L.77/1989), el (D.206/1990) y la (L.241/1995), entre otras; esto comprueba que hay una consistencia en términos jurídicos y políticos, y los detractores de la ley (L.1820/2016) simplemente están en contra o desdeñan el Acuerdo de Paz alcanzado. En realidad, esta ley tiene la virtud de caracterizarse por su claridad y detalles, lo que la hace mucho más superior que otros instrumentos de procesos de paz que se han aplicado sin mucho éxito como el M-19 en los años 90. 
Sostiene Estrada (2019) que el propósito de la mencionada ley fue reforzado con el acto legislativo 01 de 2017, destinado a crear el Sistema Integral de Verdad, Justicia, Reparación y No Repetición, incluida la Jurisdicción Especial para la Paz; y el acto legislativo 02 de 2017, concebido como un blindaje jurídico del Acuerdo de Paz; el acto legislativo 03 de 2017 elaborado para regular de forma parcial la reincorporación de las FARC a la política; y la Ley Estatuaria de la Jurisdicción Especial para la Paz (JEP) la cual tuvo un camino cuesta arriba para que pudiera ser sancionada el año pasado por el actual presidente Duque pasándose a conocer como la (L.1957/2019).

Previamente, en el 2018, se aprobó la ley (L.1922/2018), por la cual se adoptan unas reglas de procedimiento para la jurisdicción especial para la paz; la cual, junto con la ley (L.1957/2019), son consideradas normativas aprobadas más allá del marco fast track (CINEP/PPP-CERAC, 2019). Por otra parte, en cuanto a decretos con fuerza de ley, además de la ley 277, es pertinente hacer mención de otros como el Decreto (L.588/2017) destinado a organizar la Comisión para el Esclarecimiento de la Verdad, la Convivencia y la no Repetición. El 589 de 5 de abril de 2017 mediante el cual es organizada la Unidad de Búsquedas de personas desparecidas por el conflicto armado; El decreto (D.700/2017) que se refiere a la acción de habeas corpus si hay una privación de libertad indebida prolongada, que además deriva por la falta de aplicación oportuna de la ley (L.1820) y el decreto ley 277. El decreto (D.706/2017), que estable un trato especial a los miembros de la fuerza pública en razón del Sistema Integral de Verdad, Justicia, Reparación y No Repetición. El (D.775/2017), que establece normas para que el Sistema de Defensa Técnica y Especializada de los Miembros de la Fuerza Pública, preste servicios de defensa técnica a los miembros de la Fuerza Pública en el Sistema Integral de Verdad, Justicia, Reparación y No Repetición. El decreto (D.903/2017), que versa sobre el inventario de bienes y activos a disposición de las FARC.

Para el año en corrientes, es decir, 2020, de acuerdo con información suministrada por la FARC, ya como partido, han sido 222 los excombatientes que han sido asesinados desde que en el 2016 se firmara el Acuerdo de Paz. Dicho partido, al tiempo que denunciaba estos hechos, alertó que en Antioquia, una de las zonas más afectadas por la violencia en contra de los excombatientes de las FARC, al menos 25 excombatientes han sido asesinados en lo que va de 2020.(Agencia AFP, 2020). Por esta razón la JEP se ha visto obligada a dictar medidas cautelares colectivas para favorecer a los excombatientes que ahora están siendo víctimas de ataques. También se ha hecho saber que 93 antiguos rebeldes se han dado casos de desplazamiento forzoso, o que son obligados a abandonar las áreas donde desarrollan su reincorporación en un sentido social y económico. Todo lo cual constituye una violación a los derechos fundamentales, pero también atenta contra los derechos que tienen las víctimas a conocer la realidad de lo ocurrido en el conflicto, pues los antiguos guerrilleros, si cuentan la verdad, pueden aspirar a penas alternativas a la prisión, reparando a los afectados y prometiendo alejarse definitivamente de la violencia.

Esta situación pone en peligro el Acuerdo de Paz porque los exguerrilleros pueden sentirse traicionados o que simplemente están recibiendo la peor parte de un acuerdo que los ha llevado a una condición peyorativa. Incluso ya se han presentado casos de antiguos rebeldes que han declarado su intención de regresar a las armas como el caso de Francisco Durango Úsuga y otros disidentes (Semana, 2020). Más significativo ha sido el llamado de vuelta a las armas de Iván Márquez, figura protagonista en las negociaciones, alegando que el gobierno está irrespetando el Acuerdo (Casey, 2019). Sin embargo, el Acuerdo ha mostrado cierta fortaleza; se ha capturado a Durango Úsula, el Tribunal de paz los excluyó a los disidentes de los beneficios penales acordados y el partido FARC los expulsó de su organización.

\section{Conflictos de violencia en Colombia}

Justicia Vol. 25 No. 38: pp. 232-242. Julio-Diciembre, 2020. https://doi.org/10.17081/just.25.38.4485 
El Conflicto Armado en Colombia se produce de manera continua, convirtiéndole en objeto de temas centrales en diversas investigaciones, por lo que existe un amplio análisis acerca del tema, diversos expertos han aseverado sus opiniones acerca del gran conflicto que atraviesa Colombia, desde hace más de medio siglo.

Según el Comité Internacional de la Cruz Roja "En 2017, la Unidad para las Víctimas reportó cerca de 77.000 víctimas del conflicto y la violencia, la mayoría desplazados. No solo se trata de un número preocupante de personas. Cada cifra representa la historia de dolor de una familia que nunca debería haber sufrido una situación así." Por lo que este tema abarca en Colombia la primera fila en los noticieros, radios, periódicos, y otros medios de comunicación que mencionan este fenómeno como el más largo en la historia de un país, únicamente Colombia ha experimentado esto.

Por otra parte, la periodista Heidi Tamayo Ortiz, en su artículo publicado en el periódico El Mundo (2015), "Conflicto armado en Colombia: factores, actores y efectos múltiples", pretende individualizar y caracterizar el conflicto colombiano y señala de forma categórica que: "Al caracterizar el conflicto armado que ha vivido Colombia, algunos de los expertos consideran que este debe denominarse como: conflicto social armado; mientras que otros apuntan a que ha sido una guerra civil, simplemente una guerra, un conflicto irregular. Ante la diversidad de opiniones, se ha optado por nombrarlo como: Conflicto Armado Interno; un Concepto que se ha Manejado en la mesa de Negociaciones". (Tamayo, 2015, pág.3)

De igual manera, se han presentado posibles soluciones en mecanismos jurídicos interpuesto por el Gobierno nacional, en la búsqueda de una solución efectiva para lograr la ansiada paz que el pueblo quiere, tener desde el inicio mismo de su construcción como república.

“La paz será mi puerto, mi gloria, mi recompensa, mi esperanza, mi dicha y cuanto es precioso en el mundo" (Bolívar, 1842). Con esta frase alusiva a la paz se refleja el anhelo y la necesidad histórica y universal de la construcción de herramientas o fórmulas que permitan una convivencia pacífica entre las personas o grupos de una sociedad.

La anterior opinión es sustentada desde las formas de violencia que aún se presentan en la urbanidad, y que incluso llegan no solo hasta las áreas rurales, sino también en ciudades de avanzada como el caso de Barranquilla del departamento del Atlántico del territorio colombiano.

No obstante, desde la implementación del proceso de paz entre el grupo al margen de la ley (FARC) y el estado colombiano han surgido muchas incógnitas, inconformidades y apoyos a este proceso de paz por parte de la población civil. Todas estas inconformidades y apoyos al proceso de paz, entre las FARC y el Gobierno Nacional se deben a que la violencia sin lugar a dudas es un factor que por más que se trate de erradicar, siempre va a pertenecer en la memoria de quienes sufrieron las más viles consecuencias en este proceso histórico violento.

Es por estos motivos, se enfatiza que a la hora de implementar una propuesta que busque eliminar cierta parte del factor "violencia" muchos no van a estar en concordancia con las soluciones negociadas entre los bandos.

Por lo cual puede decirse que, desde el ámbito social, quienes sufrieron directamente la violencia de este conflicto armado, buscan un proceso especial que de alguna manera implemente justicia, pero una justicia que satisfaga las necesidades de ecuanimidad de éstos, y aspirando que el Estado colombiano desde su perspectiva jurídica del derecho a la paz, llegue a un común acuerdo en su mayoría, bajo el marco legal que impone la constitución a la hora de protección de derechos. 
En ese orden de ideas, una ciudadanía entendida como la condición que determina por una parte; su obediencia a la autoridad del Estado al que pertenece y, por otra, el libre ejercicio de los derechos y privilegios que éste le otorga, incluyendo en ese orden la participación ciudadana y las acciones de defensa de los derechos humanos. (Hernández, Meléndez, Chumaceiro \& Gil, 2017).

\section{Conclusiones}

El conflicto armado en Colombia ha representado un problema que dificulta el proceso de paz dentro de la nación, por la misma constitución de origen de los enfrentamientos relacionados con el tema de la tenencia de tierras y bienes que según los argumentos ideológicos han perpetuado el latifundio y su enriquecimiento selectivo, pero que en su diatriba reprodujeron la expropiación y generaron víctimas del despojo por parte de grupos delincuentes enfrentados. Víctimas no solo del desplazamiento sino de violaciones sistemáticas a sus derechos humanos, integridad y seguridad física.

En la evolución histórica del conflicto en Colombia se puede aseverar que este surgió en la lucha y protección de los bienes del campesinado de la época y que aun actualmente en pleno S. XXI., estos grupos delinquen, secuestran, apoyados con recursos económicos y extractivos desde el narcotráfico y el contrabando. La violencia ha trasegado en intereses particulares, que a pesar de todos los intentos de los gobiernos por restablecer la paz y el acuerdo con estos grupos, y de que en el año 2017 el presidente Juan Manuel Santos firmó acuerdos de paz, actualmente desde el año 2019 nuevamente están reapareciendo los grupos armados con actos de violencia y asesinatos.

Existe un marco jurídico robustecido en Colombia producto de estos procesos que han generado todo un andamiaje legal. Entre los instrumentos jurídicos del derecho a la paz en Colombia, se encuentran la Constitución, la Ley de Justicia y Paz, Ley 1152 de 2006, la ley 1182 de 2007, entre otras que buscan principalmente brindar justicia a los campesinos víctimas del desplazamiento por los grupos armados, estos tienen como función regular que se cumpla el principio de paz que establece la Constitución.

Asimismo, es necesario que el Estado en garantía de los derechos de los ciudadanos, restablezca las políticas que han promulgado en resolución al conflicto armado, en el que se deben restringir acuerdos en los que no se considere la responsabilidad penal de los que delinquen en contra de las personas y hacen aptos de violencia. El Estado debe implementar lineamientos en búsqueda de la justicia social para todos, incrementando los derechos de los trabajadores, de los campesinos y restableciendo un salario acorde a los gastos y al disfrute de su tiempo, una manera de generar calidad de vida para el bienestar general y ocupación a través del trabajo digno.

La paz, es un mecanismo de resolución de conflictos, vale la negociación, pero también se debe regular y vigilar el cumplimiento de cada compromiso de estos grupos armados, que atentan contra la seguridad de la nación, además que el marco normativo vigente necesariamente se traduzca en una aplicación eficiente, incluyente y propositiva.

\section{Referencias}

Agencia AFP. (2020) Justicia de paz en Colombia ordena proteger a excombatientes de las FARC tras 222 asesinatos. Descargado 30 de julio de 2020. En:https://www.elcomercio.com/actualidad/proteccionexcombatientes-farc-colombia-asesinatos.html

Casey, N. (2019) Iván Márquez, exlíder de las Farc, llama a volver a las armas en Colombia. Descargado 20 agosto de 2019. En: The New York Times:https://www.nytimes.com/es/2019/08/29/espanol/americalatina/colombia-farc-armas.html

Justicia Vol. 25 No. 38: pp. 232-242. Julio-Diciembre, 2020. https://doi.org/10.17081/just.25.38.4485 
Cerpa, Andreina y Molina, Olga (2019). Perspectiva histórico-jurídica del derecho a la paz en Colombia.TESIS DE GRADO con calificación Meritoria para optar al título de Abogado. Tutora: Judith Hernández García de Velazco y Cotutor: Roberto Jiménez. Programa de Derecho, Universidad de la Costa CUC. http:// repositorio.cuc.edu.co/handle/11323/6008

CINEP/PPP-CERAC (2019) Documento de balance de los primeros 30 meses de la implementación del Acuerdo Final. Bogotá: Centro de Investigación y Educación Popular.

CSJ Penal, 15 abril. 1926,Sentencia de la Sala de Negocios Generales.

Currie, Lauchlin, Bases de un programa de fomento para Colombia. Informe de una misión. Primera parte: el problema, Bogotá, Banco de la República, (1950).

Combita, Luz, Delgadillo Reina y Torres Sandra. El Posconflicto en Colombia: Una Mirada hacia los diferentes procesos de Paz. Ponencia presentada en la Conferencia regional "Globalización, Migración y Derechos Humanos", organizada por el Programa Andino de Derechos Humanos, PADH. Quito Ecuador. Septiembre 16, 17 y 18 de 2003.

Duque Mauricio. Constitución Política de Colombia actualizada, concordada y con desarrollo legislativo. Edición 2006.

Estrada, J. (2019). El acuerdo de paz en Colombia. Bogotá: Clacso

Fajardo M, D. (2015). Estudio sobre los orígenes del conflicto social armado, razones de su persistencia y sus efectos más profundos en la sociedad colombiana. Universidad Externado de Colombia. Noviembre

García Otero,R. C. (2019). Contexto de una sociedad en Conflicto: Visión panorámica de la violencia estructural en Colombia. Justicia, 24(36), 88-101. https://doi.org/10.17081/just.24.36.3524

Hernández G, J. Chumaceiro H, A. Ziritt T. G. \& Acurero, M. (2018). Culture for peace in Colombia. An approach from public policies/Cultura para la paz en Colombia. Una aproximación desde las políticas públicas. Opción, 34(86), 612-641. file:///C:/Users/CUC/Downloads/23856-37478-1-SM\%20(1).pdf

Hernández G, J. Meléndez, Y. Chumaceiro, A. y Gil, X. (2017). Cultura democrática, paz y convivencia social en Colombia. Abordaje desde una "Escuela para Ciudadanos. En el libro: Conflictos y posconflictos. Pasado y presente en América Latina y el Caribe, caso Colombia. Fondo editorial UNERMB. ISBN: 978980-427-017-8. Cabimas.

LeGrand, Catherine, Colonización y protesta campesina en Colombia 1850-1950, Universidad Nacional, Bogotá, 1988.

Ley. 975/2005, Diario Oficial 45.980 (Julio)

Ley.1922/ 2018 (Julio). Presidencia de la República

Meléndez Monroy, Y., Paternina Sierra, J., \& Velásquez Martínez, D. (2018). Procesos de paz en Colombia: derechos humanos y familias víctimas del conflicto armado. / Peace processes in Colombia: human rights and victim families of the armed conflicto. JURÍDICAS CUC, 14(1), 55-74. https://doi.org/10.17981/ juridcuc.14.1.2018.03

Mínguez, X. (2015) Conflicto y Paz en Colombia. Significados en Organizaciones Defensoras de los Derechos humanos. Descargado 12 de noviembre de 2019. En: https://scholar.google. es/scholar?start=10\&q=articulos+procesos+de+paz+en+colombia\&hl=es\&lr=lang_es\&as_ $s d t=0,5 \# d=g s \_q a b s \& u=\% 23 p \% 3 D p l v V J d d t r s U J$ 641. Maracaibo.

Narváez Mercado, B., Matzumoto Benitez, N., Mira-Olano, L., \& Zúñiga-Bolívar, A. (2019). Los derechos e inclusión de las víctimas del conflicto armado vs. las obligaciones Estado colombiano/ rights and inclusion of the victims of the armed conflict vs. the obligations of the Colombian State. JURÍDICAS CUC, 15(1), 321-352. https://doi.org/10.17981/juridcuc.15.1.2019.13 
Semana de (2020) Descargado 25 de julio de 2020. Capturan a alias Ariel o Frita, quien aparecía en el video con Iván Márquez. En:https://www.semana.com/nacion/articulo/capturan-a-alias-ariel-o-fritaquien-aparecia-en-el-video-con-ivan-marquez/689164

PNUD. Objetivos del desarrollo sostenible (2020) https://www.undp.org/content/undp/es/home/ sustainable-development-goals.html

Trejos-Rosero, L., Badillo-Sarmiento, R., \& Irreño-Quijano, Y. (2019). El caribe colombiano: entre la construcción de paz y la persistencia del conflicto I The Colombian Caribbean Region: between peacebuilding and the persistent of the violence. JURÍDICAS CUC, 15(1), 9-46. https://doi.org/10.17981/juridcuc.15.1.2019.01 